JOURNAL OF

FUNCTION SPACES AND APPLICATIONS

Volume 7, Number 1 (2009), 43-59
(C) 2009, Scientific Horizon http://www.jfsa.net

\title{
Two-weight inequalities for singular integral operators satisfying a variant of Hörmander's condition
}

\author{
Vagif S. Guliyev
}

(Communicated by Vakhtang Kokilashvili)

2000 Mathematics Subject Classification. 42B20.

Keywords and phrases. Weighted Lebesgue space, convolution operator, two-weighted inequality, Hörmander's condition.

Abstract. In this paper, we present some sufficient conditions for the boundedness of convolution operators that their kernel satisfies a certain version of Hörmander's condition, in the weighted Lebesgue spaces $L_{p, \omega}\left(\mathbb{R}^{n}\right)$.

\section{Introduction}

Let $\mathbb{R}^{n}$ be $n$-dimensional Euclidean space, $x=\left(x_{1}, \ldots, x_{n}\right), \xi=$ $\left(\xi_{1}, \ldots, \xi_{n}\right)$ are vectors in $\mathbb{R}^{n}, x \cdot \xi=x_{1} \xi_{1}+\ldots+x_{n} \xi_{n},|x|=(x \cdot x)^{1 / 2}$, $\mathbb{R}_{0}^{n}=\mathbb{R}^{n} \backslash\{0\}$.

Suppose that $\omega$ be a positive, measurable, and real function defined in $\mathbb{R}^{n}$, i.e., is a weight function. By $L_{p, \omega}\left(\mathbb{R}^{n}\right)$ we denote the space of measurable functions $f(x)$ on $\mathbb{R}^{n}$ with finite norm

$$
\|f\|_{L_{p, \omega}\left(\mathbb{R}^{n}\right)}=\left(\int_{\mathbb{R}^{n}}|f(x)|^{p} \omega(x) d x\right)^{1 / p}, 1 \leq p<\infty .
$$


For $\omega=1$, we obtain the nonweighted space $L_{p}$, i.e., $L_{p, 1}\left(\mathbb{R}^{n}\right)=L_{p}\left(\mathbb{R}^{n}\right)$.

We write $f \in L_{p}^{\text {loc }}\left(\mathbb{R}^{n}\right), 1 \leq p<\infty$, if $f$ belongs to $L_{p}(F)$ on any closed bounded set $F \subset \mathbb{R}^{n}$.

Let $K: \mathbb{R}_{0}^{n} \rightarrow \mathbb{R}, K \in L_{1}^{\text {loc }}\left(\mathbb{R}_{0}^{n}\right), \mathbb{R}_{0}^{n}=\mathbb{R}^{n} \backslash\{0\}$, be a function satisfying the following conditions:

1) $K(t x) \equiv K\left(t x_{1}, \ldots, t x_{n}\right)=t^{-n} K(x)$ for any $t>0, x \in R_{0}^{n}$;

2) $\int_{|x|=1} K(x) d \sigma(x)=0$

3) $\int_{0}^{1} \frac{w(t)}{t} d t<\infty$, where $w(t)=\sup _{|\xi-\eta| \leq t}|K(\xi)-K(\eta)|$ for $|\xi|=|\eta|=$ 1 .

Let $f \in L_{p}\left(\mathbb{R}^{n}\right), 1<p<\infty$, and consider the following singular integral (1)

$$
T f(x)=p . v \cdot \int_{\mathbb{R}^{n}} K(x-y) f(y) d y=\lim _{\varepsilon \rightarrow 0} \int_{\left\{y \in \mathbb{R}^{n}:|x-y|>\varepsilon\right\}} K(x-y) f(y) d y .
$$

In the following theorem Calderon and Zygmund [5] proved the boundedness of the operator $T$.

Theorem 1. Suppose that the kernel $K$ of the singular integral (1) satisfies conditions 1$)-3)$ and $f \in L_{p}\left(\mathbb{R}^{n}\right), 1 \leq p<\infty$. Then the singular integral exists for $x \in \mathbb{R}^{n}$ almost everywhere and the following inequalities holds

$$
\begin{aligned}
\|T f\|_{L_{p}\left(\mathbb{R}^{n}\right)} & \leq C_{1}\|f\|_{L_{p}\left(\mathbb{R}^{n}\right)}, 1<p<\infty, \\
\int_{\left\{x \in \mathbb{R}^{n}:|T f(x)|>\lambda\right\}} d x & \leq \frac{C_{2}}{\lambda} \int_{\mathbb{R}^{n}}|f(x)| d x,
\end{aligned}
$$

where $C_{1}, C_{2}>0$ is independent of $f$.

Hörmander [13] imposed a weaker constraint on the kernel of the singular integral (1), namely,

$$
\int_{\left\{x \in \mathbb{R}^{n}:|x|>2|y|\right\}}|K(x-y)-K(x)| d x \leq C,
$$

where $K \in L_{1}^{\text {loc }}\left(\mathbb{R}_{0}^{n}\right)$ and $C>0$ is a constant independent of $y$. By replacing condition 3) with condition (2), under conditions 1), 2) he proved Theorem 1 for singular integrals with kernels satisfying condition (2). This condition is related to condition 3), and under this condition, inequality (2) holds ( see [19]). 
On the other hand, singular integrals whose kernels do not satisfy Hörmander's condition (2) are widely considered, for example oscillatory and some other singular integrals ( see [20]).

Suppose that $K \in L_{2}\left(\mathbb{R}^{n}\right)$ is a function, satisfying the following conditions:

(K1) $\|\widehat{K}\|_{\infty} \leq C$;

(K2) $|K(x)| \leq \frac{C}{|x|^{n}}$

(K3) There exist functions $A_{1}, \ldots, A_{m} \in L_{1}^{\text {loc }}\left(\mathbb{R}_{0}^{n}\right)$, and the finite family $\Phi=\left\{\phi_{1}, \ldots, \phi_{m}\right\}$ of essentially bounded functions in $\mathbb{R}^{n}$ such that $\left.\mid \operatorname{det}\left[\phi_{j}\left(y_{i}\right)\right)\right]\left.\right|^{2} \in R H_{\infty}\left(R^{n m}\right), y_{i} \in \mathbb{R}^{n}, i, j=1, \ldots, m$;

(K4) For a fixed $\gamma>0$ and for any $|x|>2|y|>0$,

$$
\left|K(x-y)-\sum_{i=1}^{m} A_{i}(x) \phi_{i}(y)\right| \leq C \frac{|y|^{\gamma}}{|x-y|^{n+\gamma}},
$$

where $C>0$ is a constant and $\widehat{K}(\xi)=\int_{\mathbb{R}^{n}} e^{-i(x, \xi)} K(x) d x$ is the Fourier transform of the function $K$. In general, the functions $A_{i}$, $\phi_{i}, i=1, \ldots, m$ defined in $\mathbb{R}_{0}^{n}$ are complex-valued.

Remark 1. Any kernel satisfying condition (3) also satisfies the condition

$$
\int_{|x|>2|y|}\left|K(x-y)-\sum_{i=1}^{m} A_{i}(x) \phi_{i}(y)\right| d x \leq C,|x|>2|y| .
$$

Note that conditions $(K 1)-(K 4)$ were imposed in [20] and condition (4) was studied in [10]. For example, for $m=1, A_{1}(x)=K(x), \phi_{1}(y) \equiv 1$ condition (4) yields Hörmander's condition (2). Note that, in this sense, condition (4) is a generalization of Hörmander's condition (2).

There exist other conditions stronger than condition $(2)$ (see $[9,21])$. The function $K(x)=(\sin x) / x$ satisfies conditions $(K 1)-(K 4)$ and does not satisfy conditions 1), 2), and Hörmander's condition (2) (see [3]).

Definition 1. [17] It is said that a locally integrable weight function $\omega$ belongs to $A_{p}\left(\mathbb{R}^{n}\right)$, where $1<p<\infty$, if

$$
\sup _{B}\left(|B|^{-1} \int_{B} \omega(x) d x\right)\left(|B|^{-1} \int_{B} \omega(x)^{1-p^{\prime}} d x\right)^{p-1}<\infty,
$$

where the supremum is taken over all balls $B \subset \mathbb{R}^{n}$ and $p^{\prime}=\frac{p}{p-1}$. 
For $p=1$, we say $\omega \in A_{1}\left(\mathbb{R}^{n}\right)$, if

$$
\sup _{B}\left(|B|^{-1} \int_{B} \omega(x) d x\right) \underset{B}{\operatorname{ess} \sup } \frac{1}{\omega(x)}<\infty,
$$

or

$$
|B|^{-1} \int_{B} \omega(x) d x \leq C \omega(x) \text { a.e. } x \in B
$$

for any balls $B \subset \mathbb{R}^{n}$.

Suppose that the function $K$ satisfies conditions $(K 1)-(K 4)$. For $f \in L_{p}\left(\mathbb{R}^{n}\right), 1 \leq p<\infty$ define the following convolution operator generated by the kernel $K$ as

$$
A f(x)=\int_{\mathbb{R}^{n}} K(x-y) f(y) d y .
$$

For the convolution operator (5), the following theorem holds.

Theorem 2. [20] Suppose that $w \in A_{p}\left(\mathbb{R}^{n}\right), 1 \leq p<\infty$, and the kernel of the convolution operator (5) satisfies conditions $(K 1)-(K 4)$. Then the following inequalities holds:

$$
\begin{gathered}
\|A f\|_{L_{p, w}\left(\mathbb{R}^{n}\right)} \leq C_{3}\|f\|_{L_{p, w}\left(\mathbb{R}^{n}\right)}, 1<p<\infty, \\
\int_{\left\{x \in \mathbb{R}^{n}:|A f(x)|>\lambda\right\}} \omega(x) d x \leq \frac{C_{4}}{\lambda} \int_{\mathbb{R}^{n}}|f(x)| \omega(x) d x,
\end{gathered}
$$

where $C_{3}, C_{4}>0$ is independent of $f$.

Note that in the "nonweighted" case, when condition $(K 2)$ is not imposed and condition (3) is replaced by condition (4), Theorem 2 was proved in [10].

Lemma 1. Suppose that $1 \leq p \leq q \leq \infty$ and $u(t)$ and $v(t)$ are positive functions defined on $(0, \infty)$.

(i) For the validity of the inequality

$$
\left(\int_{0}^{\infty} u(t)\left|\int_{0}^{t} \varphi(\tau) d \tau\right|^{q} d t\right)^{1 / q} \leq K_{1}\left(\int_{0}^{\infty}|\varphi(t)|^{p} v(t) d t\right)^{1 / p}
$$

with a constant $K_{1}$, not depending on $\varphi$, it is necessary and sufficient that

$$
\sup _{t>0}\left(\int_{t}^{\infty} u(\tau) d \tau\right)^{p / q}\left(\int_{0}^{t} v(\tau)^{1-p^{\prime}} d \tau\right)^{p-1}<\infty .
$$


(ii) For the validity of the inequality

$$
\left(\int_{0}^{\infty} u(t)\left|\int_{t}^{\infty} \varphi(\tau) d \tau\right|^{q} d t\right)^{1 / q} \leq K_{2}\left(\int_{0}^{\infty}|\varphi(t)|^{p} v(t) d t\right)^{1 / p}
$$

with a constant $K_{2}$, not depending on $\varphi$, it is necessary and sufficient that

$$
\sup _{t>0}\left(\int_{0}^{t} u(\tau) d \tau\right)^{p / q}\left(\int_{t}^{\infty} v(\tau)^{1-p^{\prime}} d \tau\right)^{p-1}<\infty .
$$

Lemma 1 was established by Muckenhoupt [18] for $1 \leq p=q \leq \infty$ and J.S. Bradley [4], V.M. Kokilashvili [14], V.G. Maz'ya [16] for $p<q$.

Lemma 2. [15] Let $u(t)$ and $v(t)$ be positive functions on $(0, \infty)$.

(i) If the following condition is satisfied

$$
\sup _{t>0}\left(\int_{t}^{\infty} v(\tau) d \tau\right) \operatorname{ess~sup}_{\tau \in(0,2 t)} \frac{1}{u(\tau)}<\infty,
$$

then the inequality

$$
\int_{0}^{\infty} v(t)\left|\int_{0}^{t} F(\tau) d \tau\right| d t \leq c \int_{0}^{\infty} u(t)|F(t)| d t
$$

holds, where the constant $c>0$ does not depend on $F$.

(ii) If the following condition is satisfied

$$
\sup _{t>0}\left(\int_{0}^{t} v(\tau) d \tau\right) \underset{\tau \in\left(\frac{t}{2}, \infty\right)}{\operatorname{ess} \sup } \frac{1}{u(\tau)}<\infty
$$

then the inequality

$$
\int_{0}^{\infty} v(t)\left|\int_{t}^{\infty} F(\tau) d \tau\right| d t \leq c \int_{0}^{\infty} u(t)|F(t)| d t
$$

holds, where the constant $c>0$ does not depend on $F$.

Lemma 3. $[1,6]$ Suppose that $1 \leq p \leq q \leq \infty$ and $u(x)$ and $v(x)$ are positive functions defined on $\mathbb{R}^{n}$.

(i) For the n-dimensional Hardy inequality

$$
\left(\int_{\mathbb{R}^{n}}\left(\int_{|y|<|x| / 2}|f(y)| d y\right)^{q} \omega(x) d x\right)^{1 / q} \leq C_{5}\left(\int_{\mathbb{R}^{n}}|f(x)|^{p} v(x) d x\right)^{1 / p}
$$


with a constant $C_{5}$, independent on $f$, to hold, it is necessary and sufficient that the following condition be satisfied:

$$
\sup _{R>0}\left(\int_{|x|>2 R} \omega(x) d x\right)^{1 / q}\left(\int_{|x|<R} v^{1-p^{\prime}}(x) d x\right)^{1 / p^{\prime}}<\infty .
$$

(ii) For the n-dimensional (dual) Hardy inequality

$$
\left(\int_{\mathbb{R}^{n}}\left(\int_{|y|>2|x|}|f(y)| d y\right)^{q} u(x) d x\right)^{1 / q} \leq C_{6}\left(\int_{\mathbb{R}^{n}}|f(x)|^{p} \omega(x) d x\right)^{1 / p}
$$

with a constant $C_{6}$, independent on $f$, to hold, it is necessary and sufficient that the following condition be satisfied:

$$
\sup _{R>0}\left(\int_{|x|<R} u(x) d x\right)^{1 / q}\left(\int_{|x|>2 R} \omega^{1-p^{\prime}}(x) d x\right)^{1 / p^{\prime}}<\infty .
$$

Lemma 4. $[8,15]$ Suppose that $1 \leq p<\infty, \beta>1, \varphi \in A_{p}\left(\mathbb{R}^{n}\right)$, and suppose that $u, u_{1}$ are positive increasing (decreasing) functions defined on $(0, \infty)$. Suppose that $\omega(x)=u(|x|) \varphi(x), \omega_{1}(x)=u_{1}(|x|) \varphi(x)$ and the weighted pair $\left(\omega(x), \omega_{1}(x)\right)$ satisfies the following condition:

(i) For $1<p<\infty, \mathcal{A}_{p}\left(\omega, \omega_{1}\right)<\infty$, where

$$
\mathcal{A}_{p}\left(\omega, \omega_{1}\right):=\sup _{r>0}\left(\int_{|x|>2 r} \omega_{1}(x)|x|^{-n p} d x\right)\left(\int_{|x|<r} \omega^{1-p^{\prime}}(x) d x\right)^{p-1}
$$

(ii) For $p=1, \mathcal{A}_{1}\left(\omega, \omega_{1}\right)<\infty$, where

$$
\mathcal{A}_{1}\left(\omega, \omega_{1}\right):=\sup _{r>0}\left(\int_{|x|>2 r} \omega_{1}(x)|x|^{-n} d x\right) \underset{|x|<r}{\operatorname{ess} \sup } \frac{1}{\omega(x)}
$$

(iii) For $1<p<\infty, \mathcal{B}_{p}\left(\omega, \omega_{1}\right)<\infty$, where

$$
\mathcal{B}_{p}\left(\omega, \omega_{1}\right):=\sup _{r>0}\left(\int_{|x|<r} \omega_{1}(x) d x\right)\left(\int_{|x|>2 r} \omega^{1-p^{\prime}}(x)|x|^{-n p^{\prime}} d x\right)^{p-1}
$$

(iv) For $p=1, \mathcal{B}_{1}\left(\omega, \omega_{1}\right)<\infty$, where

$$
\mathcal{B}_{1}\left(\omega, \omega_{1}\right):=\sup _{r>0}\left(\int_{|x|<r} \omega_{1}(x) d x\right) \underset{|x|>2 r}{\operatorname{ess} \sup } \frac{1}{\omega(x)|x|^{n}}
$$


Then there exists a positive constant $C$ depending only on $p, n$ such that, for any $t>0$, the following inequality holds:

$$
u_{1}(2 t) \leq C \mathcal{A}_{p}\left(\omega, \omega_{1}\right) u(t) \quad\left(u_{1}(t / 2) \leq C \mathcal{B}_{p}\left(\omega, \omega_{1}\right) u(t)\right) .
$$

In the case $\varphi=1$ Lemma 4 was proved also in [11].

\section{Main results}

Theorem 3. Suppose that the kernel $K$ of the convolution operator (5) satisfies the conditions $(K 1)-(K 4)$ and $\phi \in A_{p}\left(\mathbb{R}^{n}\right), 1 \leq p<\infty$. If $\omega(x)=u(x) \phi(x)$ and $\omega_{1}(x)=u_{1}(x) \phi(x)$ are weight functions on $\mathbb{R}^{n}$, satisfies the conditions

$$
\mathcal{A}_{p}\left(\omega, \omega_{1}\right)<\infty, \mathcal{B}_{p}\left(\omega, \omega_{1}\right)<\infty,
$$

and there exist $b>0$ such that

$$
\sup _{|x| / 4<|y| \leq 4|x|} u_{1}(y) \leq b u(x) \text { for a.e. } x \in \mathbb{R}^{n} .
$$

Then there exists a $C_{7}>0$ such that, for any $f \in L_{p, \omega}\left(\mathbb{R}^{n}\right), 1<p<\infty$ the following inequality holds

$$
\int_{\mathbb{R}^{n}}|A f(x)|^{p} \omega_{1}(x) d x \leq C_{7} \int_{\mathbb{R}^{n}}|f(x)|^{p} \omega(x) d x .
$$

Moreover, the condition (6) can be replaced by the condition : there exist $b>0$ such that

$$
u_{1}(x)\left(\sup _{|x| / 4 \leq|y| \leq|x|} \frac{1}{u(y)}\right) \leq b \quad \text { for a.e. } x \in \mathbb{R}^{n} .
$$

Proof. For $k \in Z$ we define $E_{k}=\left\{x \in \mathbb{R}^{n}: 2^{k}<|x| \leq 2^{k+1}\right\}$, $E_{k, 1}=\left\{x \in \mathbb{R}^{n}:|x| \leq 2^{k-1}\right\}, E_{k, 2}=\left\{x \in \mathbb{R}^{n}: 2^{k-1}<|x| \leq 2^{k+2}\right\}$, $E_{k, 3}=\left\{x \in \mathbb{R}^{n}:|x|>2^{k+2}\right\}$. Then $E_{k, 2}=E_{k-1} \cup E_{k} \cup E_{k+1}$ and the multiplicity of the covering $\left\{E_{k, 2}\right\}_{k \in Z}$ is equal to 3 . 
Let $1<p<\infty$. Given $f \in L_{p, \omega}\left(\mathbb{R}^{n}\right)$, we write

$$
\begin{aligned}
|A f(x)|= & \sum_{k \in Z}|A f(x)| \chi_{E_{k}}(x) \\
\leq & \sum_{k \in Z}\left|A f_{k, 1}(x)\right| \chi_{E_{k}}(x)+\sum_{k \in Z}\left|A f_{k, 2}(x)\right| \chi_{E_{k}}(x) \\
& +\sum_{k \in Z}\left|A f_{k, 3}(x)\right| \chi_{E_{k}}(x) \\
\equiv & A_{1} f(x)+A_{2} f(x)+A_{3} f(x),
\end{aligned}
$$

where $\chi_{E_{k}}$ is the characteristic function of the set $E_{k}, f_{k, i}=f \chi_{E_{k, i}}$, $i=1,2,3$.

First we shall estimate $\left\|A_{1} f\right\|_{L_{p, \omega_{1}}}$. Note that for $x \in E_{k}, y \in E_{k, 1}$ we have $|y| \leq 2^{k-1} \leq|x| / 2$. Moreover, $E_{k} \cap \operatorname{suppf}_{k, 1}=\emptyset$ and $|x-y| \geq|x| / 2$. Hence by condition $(K 2)$

$$
\begin{aligned}
A_{1} f(x) & \leq C \sum_{k \in Z}\left(\int_{\mathbb{R}^{n}} \frac{\left|f_{k, 1}(y)\right|}{|x-y|^{n}} d y\right) \chi_{E_{k}} \\
& \leq C \int_{|y| \leq|x| / 2}|x-y|^{-n}|f(y)| d y \leq 2^{n} C|x|^{-n} \int_{|y| \leq|x| / 2}|f(y)| d y
\end{aligned}
$$

for any $x \in E_{k}$. Hence we have

$$
\int_{\mathbb{R}^{n}}\left|A_{1} f(x)\right|^{p} \omega_{1}(x) d x \leq\left(2^{n} C\right)^{p} \int_{\mathbb{R}^{n}}\left(\int_{|y|<|x| / 2}|f(y)| d y\right)^{p}|x|^{-n p} \omega_{1}(x) d x .
$$

Since $\mathcal{A}_{p}\left(\omega, \omega_{1}\right)<\infty$, the Hardy inequality

$$
\int_{\mathbb{R}^{n}} \omega_{1}(x)|x|^{-n p}\left(\int_{|y|<|x| / 2}|f(y)| d y\right)^{p} d x \leq C_{8} \int_{\mathbb{R}^{n}}|f(x)|^{p} \omega(x) d x
$$

holds and $C_{9} \leq c^{\prime} \mathcal{A}_{p}\left(\omega, \omega_{1}\right)$, where $c^{\prime}$ depends only on $n$ and $p$. In fact the condition $\mathcal{A}_{p}\left(\omega, \omega_{1}\right)<\infty$ is necessary and sufficient for the validity of this inequality (see [1], [6]). Hence, we obtain

$$
\int_{\mathbb{R}^{n}}\left|A_{1} f(x)\right|^{p} \omega_{1}(x) d x \leq C_{8} \int_{\mathbb{R}^{n}}|f(x)|^{p} \omega(x) d x .
$$

where $C_{9}$ is independent of $f$.

Next we estimate $\left\|A_{3} f\right\|_{L_{p, \omega_{1}}}$. It is easy to verify, for $x \in E_{k}, y \in E_{k, 3}$ we have $|y|>2|x|$ and $|x-y| \geq|y| / 2$. Since $E_{k} \cap \operatorname{supp}_{k, 3}=\emptyset$, for $x \in E_{k}$ 
by condition $(K 2)$ we obtain

$$
A_{3} f(x) \leq C \int_{|y|>2|x|} \frac{|f(y)|}{|x-y|^{n}} d y \leq 2^{n} C \int_{|y|>2|x|}|f(y)||y|^{-n} d y .
$$

Hence we have

$$
\int_{\mathbb{R}^{n}}\left|A_{3} f(x)\right|^{p} \omega_{1}(x) d x \leq\left(2^{n} C\right)^{p} \int_{\mathbb{R}^{n}}\left(\int_{|y|>2|x|}|f(y)||y|^{-n} d y\right)^{p} \omega_{1}(x) d x .
$$

Since $\mathcal{B}_{p}\left(\omega, \omega_{1}\right)<\infty$, the Hardy inequality

$$
\int_{\mathbb{R}^{n}}\left(\int_{|y|>2|x|}|f(y)||y|^{-n} d y\right)^{p} \omega_{1}(x) d x \leq C_{6} \int_{\mathbb{R}^{n}}|f(x)|^{p} \omega(x) d x
$$

holds and $C_{6} \leq c^{\prime \prime} \mathcal{B}_{p}\left(\omega, \omega_{1}\right)$, where $c^{\prime \prime}$ depends only on $n$ and $p$. In fact the condition $\mathcal{B}_{p}\left(\omega, \omega_{1}\right)<\infty$ is necessary and sufficient for the validity of this inequality (see [1], [6]). Hence, we obtain

$$
\int_{\mathbb{R}^{n}}\left|A_{3} f(x)\right|^{p} \omega_{1}(x) d x \leq C_{9} \int_{\mathbb{R}^{n}}|f(x)|^{p} \omega(x) d x,
$$

where $C_{9}$ is independent of $f$.

Finally, we estimate $\left\|A_{2} f\right\|_{L_{p, \omega_{1}}}$. From the $L_{p, \phi}\left(\mathbb{R}^{n}\right)$ boundedness of $T$ and condition (6) we have

$$
\begin{aligned}
\int_{\mathbb{R}^{n}}\left|A_{2} f(x)\right|^{p} \omega_{1}(x) d x & =\int_{\mathbb{R}^{n}}\left(\sum_{k \in Z}\left|A f_{k, 2}(x)\right| \chi_{E_{k}}(x)\right)^{p} \omega_{1}(x) d x \\
& =\int_{\mathbb{R}^{n}}\left(\sum_{k \in Z}\left|A f_{k, 2}(x)\right|^{p} \chi_{E_{k}}(x)\right) \omega_{1}(x) d x \\
& =\sum_{k \in Z} \int_{E_{k}}\left|A f_{k, 2}(x)\right|^{p} u_{1}(x) \phi(x) d x \\
& \leq \sum_{k \in Z} \sup _{x \in E_{k}} u_{1}(x) \int_{\mathbb{R}^{n}}\left|A f_{k, 2}(x)\right|^{p} \phi(x) d x \\
& \leq\|A\|_{\phi}^{p} \sum_{k \in Z} \sup _{x \in E_{k}} u_{1}(x) \int_{\mathbb{R}^{n}}\left|f_{k, 2}(x)\right|^{p} \phi(x) d x \\
& =\|A\|_{\phi}^{p} \sum_{k \in Z} \sup _{y \in E_{k}} u_{1}(y) \int_{E_{k, 2}}|f(x)|^{p} \phi(x) d x,
\end{aligned}
$$


where $\|A\|_{\phi} \equiv\|A\|_{L_{p, \phi} \rightarrow L_{p, \phi}}$. Since $2^{k-1}<|x| \leq 2^{k+2}, x \in E_{k, 2}$, we have by condition $(a)$

$$
\sup _{y \in E_{k}} u_{1}(y)=\sup _{2^{k-1}<|y| \leq 2^{k+2}} u_{1}(y) \leq \sup _{|x| / 4<|y| \leq 4|x|} u_{1}(y) \leq b u(x)
$$

for almost all $x \in E_{k, 2}$. Therefore we get

$$
\begin{aligned}
\int_{\mathbb{R}^{n}}\left|A_{2} f(x)\right|^{p} \omega_{1}(x) d x & \leq\|A\|_{\phi}^{p} b \sum_{k \in Z} \int_{E_{k, 2}}|f(x)|^{p} u(x) \phi(x) d x \\
& \leq C_{10} \int_{\mathbb{R}^{n}}|f(x)|^{p} \omega(x) d x
\end{aligned}
$$

since the multiplicity of covering $\left\{E_{k, 2}\right\}_{k \in Z}$ is equal to 3 , where $C_{10}=$ $3\|A\|_{\phi}^{p} b$.

Inequalities (8), (9), (10), (11) imply (7) which completes the proof.

Analogously proved the following theorem.

Theorem 4. Suppose that the kernel $K$ of the convolution operator (5) satisfies the conditions $(K 1)-(K 4)$, and $\omega(x)=u(x) \phi(x), \omega_{1}(x)=$ $u_{1}(x) \phi(x)$ are weight functions on $\mathbb{R}^{n}, \phi \in A_{1}\left(\mathbb{R}^{n}\right)$. If the weighted pair $\left(\omega(x), \omega_{1}(x)\right)$ satisfies condition (6) and

$$
\begin{aligned}
& \mathcal{A}_{1}\left(\omega, \omega_{1}\right) \equiv \sup _{r>0}\left(\int_{|x|>2 r} \omega_{1}(x)|x|^{-n} d x\right) \underset{|x|<r}{\operatorname{ess} \sup } \frac{1}{\omega(x)}<\infty \\
& \mathcal{B}_{1}\left(\omega, \omega_{1}\right) \equiv \sup _{r>0}\left(\int_{|x|<r} \omega_{1}(x) d x\right) \underset{|x|>2 r}{\operatorname{ess} \sup } \frac{1}{\omega(x)|x|^{n}}<\infty .
\end{aligned}
$$

Then there exists a $C_{11}>0$ such that, for any $f \in L_{1, \omega}\left(\mathbb{R}^{n}\right)$, the following inequality holds

$$
\int_{\left\{x \in \mathbb{R}^{n}:|A f(x)|>\lambda\right\}} \omega_{1}(x) d x \leq \frac{C_{11}}{\lambda} \int_{\mathbb{R}^{n}}|f(x)| \omega(x) d x .
$$

Theorem 5. Suppose that the kernel $K$ of the convolution operator (5) satisfies the conditions $(K 1)-(K 4)$, and $\varphi \in A_{1}\left(\mathbb{R}^{n}\right)$. Let $u$ and $u_{1}$ are positive increasing functions on $(0, \infty)$, such that the weights functions $\omega(x)=u(|x|) \varphi(x)$ and $\omega_{1}(x)=u_{1}(|x|) \varphi(x)$ satisfy the condition

$$
\mathcal{A}_{1}\left(\omega, \omega_{1}\right)<\infty
$$

Then inequality (12) is valid. 
Proof. Suppose that $f \in L_{1, \omega}\left(\mathbb{R}^{n}\right)$. Let $u_{1}$ are positive increasing functions on $(0, \infty)$ and $\mathcal{A}_{1}\left(\omega, \omega_{1}\right)<\infty$.

Without loss of generality we can suppose that $u_{1}$ may be represented by

$$
u_{1}(t)=u_{1}(0+)+\int_{0}^{t} \psi(\tau) d \tau
$$

where $u_{1}(0+)=\lim _{t \rightarrow 0} u_{1}(t)$ and $u_{1}(t) \geq 0$ on $(0, \infty)$. In fact there exists a sequence of increasing absolutely continuous functions $\varpi_{n}$ such that $\varpi_{n}(t) \leq \omega_{1}(t)$ and $\lim _{n \rightarrow \infty} \varpi_{n}(t)=\omega_{1}(t)$ for any $t \in(0, \infty)$ ( see $[2,11,7,8,12]$ for details).

We have

$$
\begin{aligned}
\int_{\left\{x \in \mathbb{R}^{n}:|A f(x)|>\lambda\right\}} \omega_{1}(x) d x= & u_{1}(0+) \int_{\left\{x \in \mathbb{R}^{n}:|A f(x)|>\lambda\right\}} \phi(x) d x \\
& +\int_{\left\{x \in \mathbb{R}^{n}:|A f(x)|>\lambda\right\}}\left(\int_{0}^{|x|} \psi(\tau) d \tau\right) \phi(x) d x \\
= & J_{1}+J_{2} .
\end{aligned}
$$

If $u_{1}(0+)=0$, then $J_{1}=0$. If $u_{1}(0+) \neq 0$ by the weak $L_{1}$ boundedness of $A, \phi \in A_{1}\left(\mathbb{R}^{n}\right)$ thanks to Lemma 4

$$
\begin{aligned}
J_{1} & \leq \frac{1}{\lambda}\|A\|_{\phi} u_{1}(0+) \int_{\mathbb{R}^{n}}|f(x)| \phi(x) d x \\
& \leq \frac{1}{\lambda}\|A\|_{\phi} \int_{\mathbb{R}^{n}}|f(x)| u_{1}(|x|) \phi(x) d x \\
& \leq \frac{b}{\lambda}\|A\|_{\phi} \int_{\mathbb{R}^{n}}|f(x)| \omega(x) d x .
\end{aligned}
$$

After changing the order of integration in $J_{2}$ we have

$$
\begin{aligned}
J_{2}= & \int_{0}^{\infty} \psi(t)\left(\int_{|x|>t} \chi\{x:|A f(x)|>\lambda\} \phi(x) d x\right) d t \\
\leq & \int_{0}^{\infty} \psi(t)\left(\int_{|x|>t} \chi\left\{x:\left|A\left(f \chi_{\{|y|>t / 2\}}\right)(x)\right|>\lambda\right\} \phi(x) d x\right. \\
& \left.+\int_{|x|>t} \chi\left\{x:\left|A\left(f \chi_{\{|y| \leq t / 2\}}\right)(x)\right|>\lambda\right\} \phi(x) d x\right) d t \\
= & J_{21}+J_{22} .
\end{aligned}
$$


Using the weak $L_{1}$ boundeedness of $A$ and Lemma 4 we have

$$
\begin{aligned}
J_{21} & \leq \frac{\|A\|}{\lambda} \int_{0}^{\infty} \psi(t)\left(\int_{|y|>t / 2}|f(y)| \phi(y) d y\right) d t \\
& =\frac{\|A\|}{\lambda} \int_{\mathbb{R}^{n}}|f(y)|\left(\int_{0}^{2|y|} \psi(t) d t\right) \phi(y) d y \\
& \leq \frac{\|A\|}{\lambda} \int_{\mathbb{R}^{n}}|f(y)| u_{1}(2|y|) \phi(y) d y \\
& \leq b \frac{\|A\|}{\lambda} \int_{\mathbb{R}^{n}}|f(y)| \omega(y) d y .
\end{aligned}
$$

Let us estimate $J_{22}$. For $|x|>t$ and $|y| \leq t / 2$ we have $|x| / 2 \leq|x-y| \leq$ $3|x| / 2$, and so

$$
\begin{aligned}
J_{22} & \leq c_{4} \int_{0}^{\infty} \psi(t)\left(\int_{|x|>t} \chi\left\{y: \int_{|y| \leq t / 2}|f(y)||x-y|^{-n} d y>\lambda\right\} \phi(x) d x\right) d t \\
& \leq c_{5} \int_{0}^{\infty} \psi(t) \chi\left\{y: \int_{|y| \leq t / 2}|f(y)||y|^{-n} d y>\lambda\right\}\left(\int_{|x|>t} \phi(x)|x|^{-n} d x\right) d t \\
& =\frac{c_{6}}{\lambda} \int_{0}^{\infty} \psi(t)\left(\int_{|x|>t} \phi(x)|x|^{-n} d x\right)\left(\int_{|y| \leq t / 2}|f(y)| d y\right) d t .
\end{aligned}
$$

The Hardy inequality

$$
\int_{0}^{\infty} \psi(t)\left(\int_{|y| \leq t / 2}|f(y)| d y\right) d t \leq C \int_{\mathbb{R}^{n}}|f(y)| \omega(|y|) d y
$$

for $p=1$ is characterized by the condition $C \leq c^{\prime} \mathcal{A}_{1}^{\prime}$ (see [4], [14]), where

$$
\begin{aligned}
\mathcal{A}_{1}^{\prime} & \equiv \sup _{r>0}\left(\int_{2 r}^{\infty}\left(\int_{|x|>t} \phi(x)|x|^{-n} d x\right) \psi(t) d t\right) \underset{\substack{\operatorname{ess} \sup \\
|x|<r}}{ } \frac{1}{\omega(x)} \\
& =\sup _{r>0}\left(\int_{|x|>2 r} \phi(x)|x|^{-n}\left(\int_{2 r}^{|x|} \psi(t) d t\right) d x\right) \underset{|x|<r}{\operatorname{ess} \sup } \frac{1}{\omega(x)} \\
& \leq \sup _{r>0}\left(\int_{|x|>2 r} \phi(x)|x|^{-n} u_{1}(|x|) d x\right) \underset{|x|<r}{\operatorname{ess} \sup } \frac{1}{\omega(x)} \\
& =\sup _{r>0}\left(\int_{|x|>2 r} \omega_{1}(|x|)|x|^{-n} d x\right) \underset{|x|<r}{\operatorname{ess} \sup } \frac{1}{\omega(x)}=\mathcal{A}_{1}\left(\omega, \omega_{1}\right)<\infty .
\end{aligned}
$$


Hence, applying the Hardy inequality, we obtain

$$
J_{22} \leq \frac{C_{12}}{\lambda} \int_{\mathbb{R}^{n}}|f(x)| \omega(|x|) d x .
$$

Combining the estimates of $J_{1}$ and $J_{2}$, we get (12) for $\omega_{1}(t)=\omega_{1}(0+)+$ $\int_{0}^{t} \psi(\tau) d \tau$. By Fatou's theorem on passing to the limit under the Lebesgue integral sign, this implies (12). The theorem is proved.

Analogously proved the following theorem.

Theorem 6. Suppose that $1<p<\infty$, the kernel $K$ of the convolution operator (5) satisfies the conditions $(K 1)-(K 4)$ and $\varphi \in A_{p}\left(\mathbb{R}^{n}\right)$. Let $u, u_{1}$ are positive increasing functions on $(0, \infty), \omega(x)=u(|x|) \varphi(x)$, $\omega_{1}(x)=u_{1}(|x|) \varphi(x)$ and $\mathcal{A}_{p}\left(\omega, \omega_{1}\right)<\infty$. Then inequality $(7)$ is valid.

Theorem 7. Suppose that the kernel $K$ of the convolution operator (5) satisfies the conditions $(K 1)-(K 4)$ and $\varphi \in A_{1}\left(\mathbb{R}^{n}\right)$. Let $u$ and $u_{1}$ are positive decreasing functions on $(0, \infty)$, such that the weights functions $\omega(x)=u(|x|) \varphi(x)$ and $\omega_{1}(x)=u_{1}(|x|) \varphi(x)$ satisfy the condition

$$
\mathcal{B}_{1}\left(\omega, \omega_{1}\right)<\infty
$$

Then inequality (12) is valid.

Proof. Without loss of generality we can suppose that $\omega_{1}$ may be represented by

$$
\omega_{1}(t)=\omega_{1}(+\infty)+\int_{t}^{\infty} \psi(\tau) d \tau
$$

where $\omega_{1}(+\infty)=\lim _{t \rightarrow \infty} \omega_{1}(t)$ and $\omega_{1}(t) \geq 0$ on $(0, \infty)$. In fact there exists a sequence of decreasing absolutely continuous fuctions $\varpi_{n}$ such that $\varpi_{n}(t) \leq \omega_{1}(t)$ and $\lim _{n \rightarrow \infty} \varpi_{n}(t)=\omega_{1}(t)$ for any $t \in(0, \infty)$ ( see $[2,11,7,8,12]$ for details).

We have

$$
\begin{aligned}
\int_{\left\{x \in \mathbb{R}^{n}:|A f(x)|>\lambda\right\}} \omega_{1}(x) d x= & u_{1}(+\infty) \int_{\left\{x \in \mathbb{R}^{n}:|A f(x)|>\lambda\right\}} \phi(x) d x \\
& +\int_{\left\{x \in \mathbb{R}^{n}:|A f(x)|>\lambda\right\}}\left(\int_{|x|}^{\infty} \psi(\tau) d \tau\right) \phi(x) d x \\
= & I_{1}+I_{2} .
\end{aligned}
$$


If $u_{1}(+\infty)=0$, then $I_{1}=0$. If $u_{1}(+\infty) \neq 0$, by the weak $L_{1}$ boundedness of $A, \phi \in A_{1}\left(\mathbb{R}^{n}\right)$ thanks to Lemma 4

$$
\begin{aligned}
J_{1} & \leq \frac{1}{\lambda}\|A\|_{\phi} u_{1}(+\infty) \int_{\mathbb{R}^{n}}|f(x)| \phi(x) d x \\
& \leq \frac{1}{\lambda}\|A\|_{\phi} \int_{\mathbb{R}^{n}}|f(x)| u_{1}(|x|) \phi(x) d x \\
& \leq \frac{b}{\lambda}\|A\|_{\phi} \int_{\mathbb{R}^{n}}|f(x)| \omega(|x|) d x .
\end{aligned}
$$

After changing the order of integration in $J_{2}$ we have

$$
\begin{aligned}
J_{2}= & \int_{0}^{\infty} \psi(t)\left(\int_{|x|<t} \chi\{x:|A f(x)|>\lambda\} \phi(x) d x\right) d t \\
\leq & \int_{0}^{\infty} \psi(t)\left(\int_{|x|<2 t} \chi\left\{x:\left|A\left(f \chi_{\{|y|>t / 2\}}\right)(x)\right|>\lambda\right\} \phi(x) d x\right. \\
& \left.+\int_{|x|<t} \chi\left\{x:\left|A\left(f \chi_{\{|y| \leq 2 t\}}\right)(x)\right|>\lambda\right\} \phi(x) d x\right) d t \\
= & I_{21}+I_{22} .
\end{aligned}
$$

Using the weak $L_{1}$ boundedness of $A$ and Lemma 4 we obtain

$$
\begin{aligned}
I_{21} & \leq\|A\| \int_{0}^{\infty} \psi(t)\left(\int_{|x|<2 t}|f(x)| \phi(x) d x\right) d t \\
& =\|A\| \int_{\mathbb{R}^{n}}|f(x)| \phi(x)\left(\int_{|x| / 2}^{\infty} \psi(t) d t\right) d x \\
& \leq\|A\| \int_{\mathbb{R}^{n}}|f(x)| u_{1}(|x| / 2) \phi(x) d x \\
& \leq b\|A\| \int_{\mathbb{R}^{n}}|f(x)| u(|x|) \phi(x) d x \\
& =b\|A\| \int_{\mathbb{R}^{n}}|f(x)| \omega(x) d x .
\end{aligned}
$$


Let us estimate $J_{22}$. For $|x|<t$ and $|y| \geq 2 t$ we have $|y| / 2 \leq|x-y| \leq$ $3|y| / 2$, and so

$$
\begin{aligned}
I_{22} & \leq c_{8} \int_{0}^{\infty} \psi(t)\left(\int_{|x|<t} \chi\left\{y: \int_{|y| \geq 2 t}|f(y)||x-y|^{-n} d y>\lambda\right\} \phi(x) d x\right) d t \\
& \leq c_{9} \int_{0}^{\infty} \psi(t) \chi\left\{y: \int_{|y| \geq 2 t}|f(y)||y|^{-n} d y>\lambda\right\}\left(\int_{|x|<t} \phi(x) d x\right) d t \\
& =\frac{c_{9}}{\lambda} \int_{0}^{\infty} \psi(t)\left(\int_{|x|<t} \phi(x) d x\right)\left(\int_{|y| \geq 2 t}|f(y)||y|^{-n} d y\right) d t .
\end{aligned}
$$

The Hardy inequality

$$
\int_{0}^{\infty} \psi(t)\left(\int_{|y| \geq 2 t}|y|^{-n}|f(y)| d y\right) d t \leq C \int_{\mathbb{R}^{n}}|f(y)| \omega(|y|) d y
$$

for $p=1$ is characterized by the condition $C \leq c^{\prime} \mathcal{B}^{\prime}{ }_{1}$ (see [4], [14]), where

$$
\begin{aligned}
\mathcal{B}^{\prime}{ }_{1} & \equiv \sup _{r>0}\left(\int_{0}^{r}\left(\int_{|x|<t} \phi(x) d x\right) \psi(t) d t\right) \underset{|x|>2 r}{\operatorname{ess} \sup } \frac{1}{\omega(x)} \\
& =\sup _{r>0}\left(\int_{|x|<r} \phi(x)\left(\int_{|x|}^{r} \psi(t) d t\right) d x\right) \underset{|x|>2 r}{\operatorname{ess} \sup } \frac{1}{\omega(x)} \\
& \leq \sup _{r>0}\left(\int_{|x|<r} \phi(x) u_{1}(|x|) d x\right) \underset{|x|>2 r}{\operatorname{ess} \sup } \frac{1}{\omega(x)} \\
& =\sup _{r>0}\left(\int_{|x|<r} \omega_{1}(|x|) d x\right) \operatorname{ess} \sup \frac{1}{\omega(x)}<\infty .
\end{aligned}
$$

Condition $\left(c^{\prime}\right)$ of the theorem guarantees that $\mathcal{B}^{\prime} \leq \mathcal{B}<\infty$. Hence, applying the Hardy inequality, we obtain

$$
I_{22} \leq \frac{C_{13}}{\lambda} \int_{\mathbb{R}^{n}}|f(x)| \omega(|x|) d x .
$$

Combining the estimates of $I_{1}$ and $I_{2}$, we get (12) for $\omega_{1}(t)=\omega_{1}(+\infty)+$ $\int_{t}^{\infty} \psi(t) d t$. By Fatou's theorem on passing to the limit under the Lebesgue integral sign, this implies (12). The theorem is proved.

Analogously proved the following theorem.

Theorem 8. Suppose that $1<p<\infty$, the kernel $K$ of the convolution operator (5) satisfies the conditions $(K 1)-(K 4)$ and $\varphi \in A_{p}\left(\mathbb{R}^{n}\right)$. Suppose 
that $u, u_{1}$ are positive decreasing functions on $(0, \infty), \omega(x)=u(|x|) \varphi(x)$, $\omega_{1}(x)=u_{1}(|x|) \varphi(x)$ and $\mathcal{B}_{p}\left(\omega, \omega_{1}\right)<\infty$. Then inequality (7) is valid.

Remark 2. Note that for the case in which $u=u_{1}=1$, Theorem 3 was proved in [20] by using different methods. Further, in the case $1<p<\infty$ Theorems 6 and 8 was proved in [3].

Acknowledgements. The author is partially supported by the grant of the Azerbaijan-U. S. Bilateral Grants Program II (project ANSF Award / 16071) and by TUBITAK (2221 visiting scientists fellowship programme, no. 220.01-619-48891. The author would like to express his thanks to Prof. V.M. Kokilashvili for many helpful discussions about this subject.

\section{References}

[1] E. Adams, On weighted norm inequalities for the Riesz transforms of functions with vanishing moments, Studia Math., 78 (1984), 107-153.

[2] I.A. Aliev and A.D. Gadjiev, Weighted estimates of multidimensional singilars generated by the generalized shift operator, English, translated into Russian Acad. Sci. Sb. Math., 77 (1994), 37-55.

[3] R.A. Bandaliyev, Two-weight inequalities for convolution operators in Lebesgue space. Mathematical Notes, 80 (2006), 3-10.

[4] J.S. Bradley, The Hardy's inequalities with mixed norms, Canad. Math. Bull., 21 (1978), 405-408.

[5] A.P. Calderon, A. Zygmund, On the existence of certain singular integrals, Acta. Math., 88 (1952), 85-139.

[6] D. Edmunds, P. Gurka and L. Pick, Compactness of Hardy-type integral operators in weighted Banach function spaces, Studia Math., 109 (1994), 73-90.

[7] D. Edmunds and V. Kokilashvili, Two-weight inequalities for singular integrals, Canad. Math. Bull., 38 (1995), 119-125.

[8] D. Edmunds, V. Kokilashvili, and A. Meskhi, Bounded and Compact Integral Operators, Kluwer, Dordrecht, Boston, London, 2002.

[9] J. Garcia-Cuerva and J.L. Rubio de Francia, Weighted Norm Inequalities and Related Topics, North- Holland Math. Studies 116, Amsterdam, 1985.

[10] D.J. Grubb and C.N. Moore, A variant of Hörmander condition for singular integrals, Colloq. Math., 73 (1997), 165-172. 
[11] V.S. Guliyev, Two-weighted inequalities for integral operators in $L_{p^{-}}$ spaces, and applications, Trudy Math. Inst. Steklov, 204 (1993), 113136. English trans. in Proc. Steklov Inst. Math., 204 (1994), 97-116.

[12] E.G. Guseinov, Singular integrals in spaces of functions that are summable with a monotone weight,(Russian) Mat. Sb. (N.S.) 132(174) (1987), 28-44, 142; translation in Math. USSR-Sb. 60 (1988), 29-46

[13] L. Hörmander, Estimates for translation invariant operators in $L^{p}$ spaces, Acta. Math., 104 (1960), 93-140.

[14] V.M. Kokilashvili, On Hardy's inequalities in weighted spaces, (Russian) Bull. Acad. Sci. Georgian SSR, 96 (1979), 37-40.

[15] V.M. Kokilashvili and A. Meskhi, Two-weight inequalities for singular integrals defined on homogeneous groups, Proc. A. Razmadze Math.Inst., 112 (1997), 57-90.

[16] V.G. Maz'ya, Einbettingssatze fur Sobolewsche Raume I, Leipzig, Teubner, 1979.

[17] B. Muckenhoupt, Weighted norm inequalities for Hardy maximal functions, Trans. Amer. Math. Soc., 165 (1972), 207-226.

[18] B. Muckenhoupt, Hardy's inequality with weights, Studia Math., 44 (1972), 31-38.

[19] E.M. Stein, Singular Integrals and Differentiability Properties of Functions, Princeton Univ. Press, Princeton, NJ, 1970.

[20] R. Trujillo-Gonzalez, Weighted norm inequalities for singular integrals operators satisfying a variant of Hörmander condition, Comment. Math. Univ. Carolin., 44 (2003), 137-152.

[21] D.K. Watson, Weighted estimates for singular integrals via Fourier transform estimates, Duke Math. J., 60 (1990), 389-399.

Department of Mathematical Analysis

Baku State University

Institute of Mathematics and Mechanics, Baku

Azerbaijan

(E-mail : vagif@guliyev.com) 


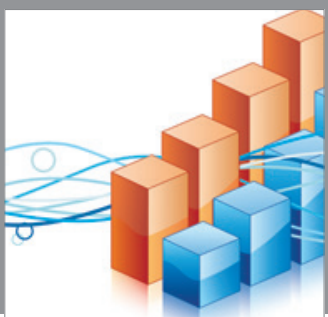

Advances in

Operations Research

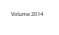

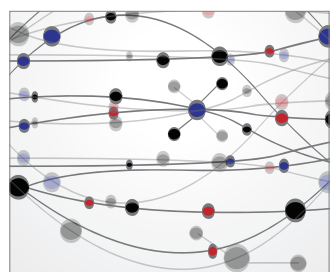

\section{The Scientific} World Journal
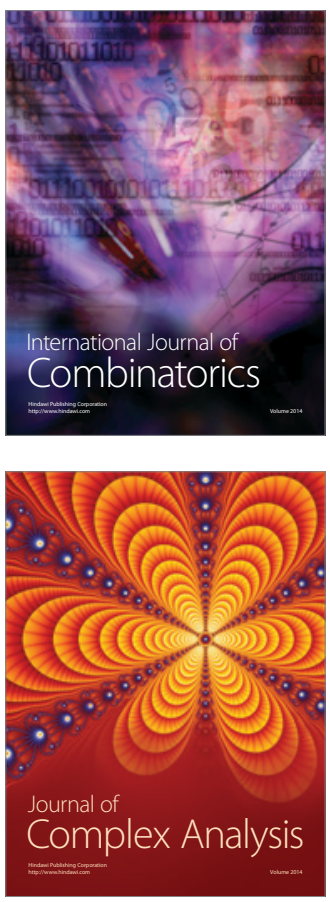

International Journal of

Mathematics and

Mathematical

Sciences
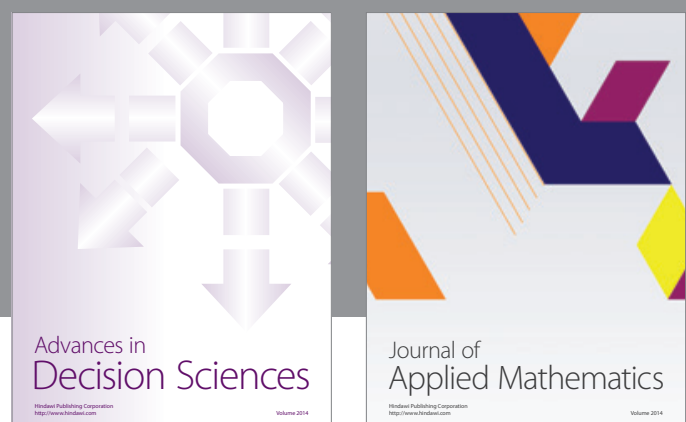

Journal of

Applied Mathematics
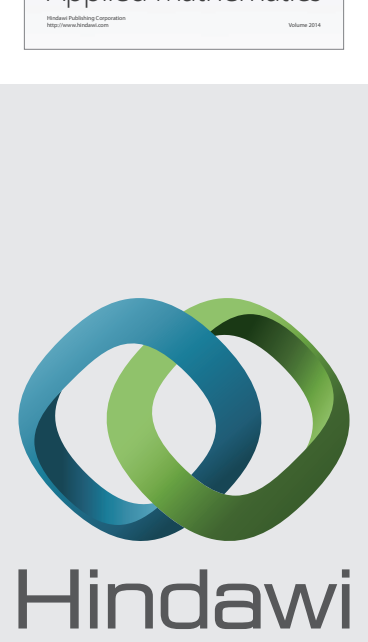

Submit your manuscripts at http://www.hindawi.com
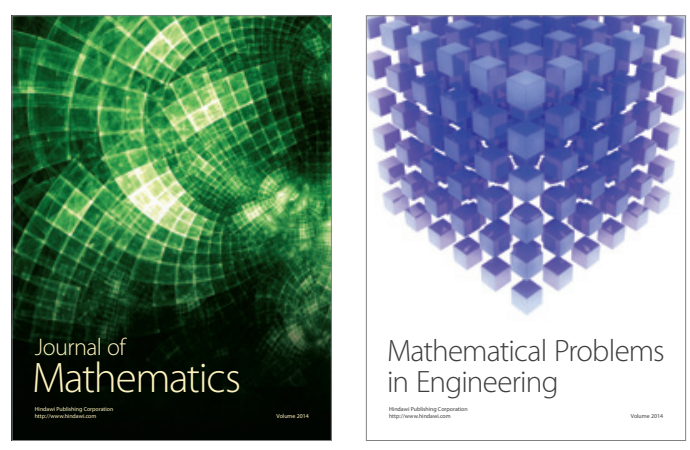

Mathematical Problems in Engineering
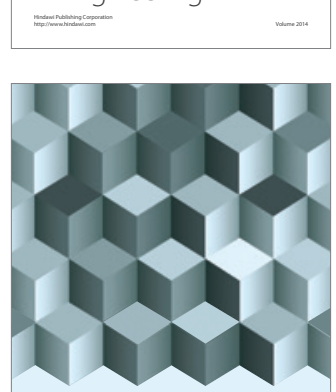

Journal of

Function Spaces
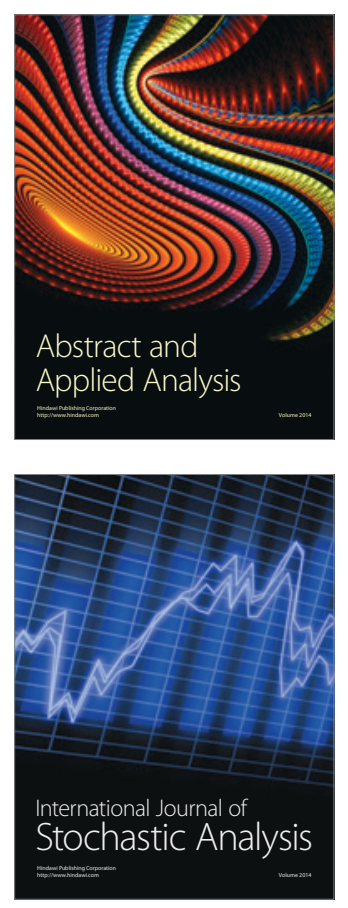

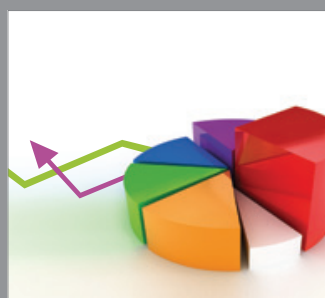

ournal of

Probability and Statistics

Promensencen
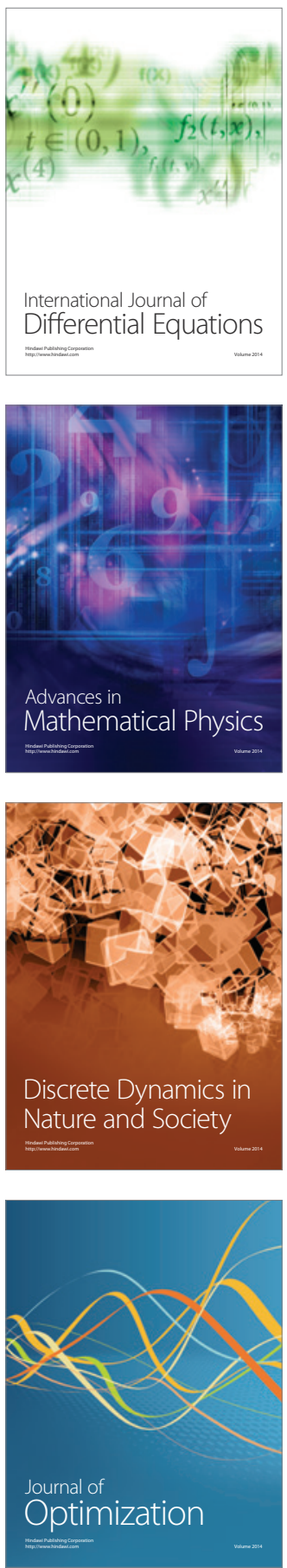